Revista Brasileira de Agricultura Irrigada v.12, nº.4, p. 2725 - 2730, 2018

ISSN 1982-7679 (On-line)

Fortaleza, CE, INOVAGRI - http://www.inovagri.org.br

DOI: $10.7127 /$ rbai.v12n400959

Protocolo 959.18 - 01/06/2018 Aprovado em 04/06/2018

\title{
ESTATÍSTICA MULTIVARIADA COMO FERRAMENTA DESCRITIVA NA ANÁLISE SENSORIAL DE ALFACE HIDROPÔNICA PRODUZIDA COM ÁGUAS SALOBRAS
}

Paula Carneiro Viana ${ }^{1}$, Fabio Tayrone Oliveira de Freitas ${ }^{2}$, Neilon Duarte da Silva ${ }^{3}$, Tales Miler Soares ${ }^{4}$, Manoela Guimarães Ferreira da $\mathrm{Paz}^{5}$

\section{RESUMO}

Plantas de alface cv. "Robusta" foram cultivadas com o objetivo de avaliar seu nível de aceitação quando produzidas em sistema hidropônico com a utilização de água doce e salobra sob diferentes frequências de recirculação da solução nutritiva, utilizando-se estatística multivariada como ferramenta descritiva. O experimento foi conduzido em blocos casualizados com cinco repetições, totalizando 30 parcelas. Foram empregadas duas qualidades de água em termos de condutividade elétrica (CEa): uma de abastecimento local (CEa 0,3 dS m m $^{-1}$ ) e outra obtida a partir da adição de $\mathrm{NaCl}$ (CEa 5,3 dS m m $^{-1}$ ), o sistema hidropônico utilizado foi do tipo DFT, com três frequências de recirculação: 0,25 h; 2 h; e 4 h. O tipo de água e a frequência de recirculação da solução nutritiva não interferiu na aceitação da alface produzida em sistema hidropônico DFT. Os consumidores de alface comprariam dependendo da aparência e textura do produto.

Palavras-chave: frequência de recirculação, salinidade, sistema NFT, solução nutritiva.

\section{MULTIVARIATE STATISTICS AS A DESCRIPTIVE TOOL IN THE SENSORY ANALYSIS OF HYDROPONIC LETTUCE PRODUCED WITH BRACKISH WATER}

\begin{abstract}
Lettuce plants cv. "Robusta" were cultivated with the objective of evaluating their level of acceptance when produced in a hydroponic system with the use of fresh and brackish water under different recirculation frequencies of the nutrient solution, using multivariate statistics as a descriptive tool. The experiment was conducted in a randomized block with five replicates, totaling 30 plots. Two water qualities were used in terms of electrical conductivity (CEa): one
\end{abstract}

1 Doutora em Engenharia Agrícola, NEAS/CCAAB/UFRB. Cruz das Almas - Bahia. Email: pcvengenharia@gmail.com;

${ }^{2}$ Mestrando em Engenharia Agrícola, UFRB, Cruz das Almas - Bahia, E-mail: oibaf.freitas@hotmail.com;

${ }^{3}$ Doutorando em Engenharia Agrícola, UFRB, Cruz das Almas - Bahia, E-mail: neylon_duart@hotmail.com;

${ }^{4}$ Prof. Doutor, NEAS/CCAAB/UFRB, Cruz das Almas - Bahia. E-mail:talesmiler@gmail.com;

${ }^{5}$ Acadêmica em Agronomia, UFRB, Cruz das Almas, BA. Email: manoelagfpaz@gmail.com. 
of the local supply (CEa $0,3 \mathrm{dS} \mathrm{m}^{-1}$ ) and the other one obtained from the addition of $\mathrm{NaCl}$ (CEa $5,3 \mathrm{dS} \mathrm{m}^{-1}$ ), The hydroponic system used was of the DFT type, with three recirculation frequencies: $0.25 \mathrm{~h}$; $2 \mathrm{~h}$; and $4 \mathrm{~h}$. The type of water and the frequency of recirculation of the nutrient solution did not interfere in the acceptance of the lettuce produced in hydroponic DFT system. Lettuce consumers would buy depending on the appearance and texture of the product.

Keywords: frequency of recirculation, salinity, NFT system, nutrient solution

\section{INTRODUÇÃO}

Atualmente várias pesquisas têm sido levantadas a respeito do uso de águas salobras em hidroponia. Tais pesquisas são estratégicas para o aproveitamento racional das águas encontradas nos poços perfurados no Semiárido ou a água de rejeito dos dessalinizadores, pois frequentemente essas têm níveis de salinidade que comprometem seu uso direto na agricultura convencional.

Dentre os vários tipos de sistemas hidropônicos, destacam-se como o mais viável em instalações comerciais o sistema NFT (Técnica do Fluxo Laminar de Nutrientes), no qual é muito usual adotar irrigações de $0,25 \mathrm{~h}$ a cada 0,25 h de intervalo. Em função da recirculação da solução, da alta frequência de irrigação, mantêm-se as raízes sempre bem supridas de água e nutrientes. Por outro lado, segundo Cova et al., (2017), o sistema DFT(Técnica do Fluxo Profundo) permite a adoção de diferentes frequências de recirculação, em virtude das raízes das plantas serem mantidas em contato contínuo com a lâmina de solução nutritiva (SN).

A análise sensorial é utilizada como método de avaliação de aceitação de um determinado produto, análise esta que é feita por meio dos sentidos humanos. $\mathrm{O}$ estabelecimento do perfil sensorial de produtos alimentares é uma atividade bastante difundida, quando se objetiva principalmente definir a aceitação do produto no mercado. Diante de um mercado consumidor cada vez mais exigente, torna-se cada vez mais importante produzir produtos de qualidade. Isso envolve produtos frescos como a alface, cujo consumo ocorre in natura, além de ser considerada a folhosa de maior consumo mundial (Krause-Sakate et al., 2008).

A análise dos dados de uma avaliação sensorial pode ser realizada, tanto pela forma unidimensional (teste de médias ou estatística descritiva) e de forma multidimensional (análise de componentes principais) (Pagè; Husson, 2001). Os métodos analíticos multidimensionais fornecem uma representação de todos os produtos no espaço podendo apresentar geralmente duas ou três dimensões. A análise fatorial mais realizada com frequência é a Análise de Componentes Principais (ACP).

Diante do exposto, objetivou-se com esse estudo avaliar a preferência e intenção de compra da alface produzida em sistema hidropônico com a utilização de água doce e salobra sob diferentes frequências de recirculação da solução nutritiva, utilizando-se estatística multivariada como ferramenta descritiva

\section{MATERIAL E MÉTODOS}

O experimento foi conduzido de 25 de fevereiro a 25 de março de 2015 no verão, em ambiente protegido, no município de Cruz das Almas - BA $\left(12^{\circ} 40^{\prime} 19^{\prime \prime} S, 3^{\circ} 6^{\prime} 23^{\prime \prime} \mathrm{W}\right)$ e altitude de 226 metros. Segundo a classificação de Köppen (1948), com a então modificação proposta por Alvares et al., (2013), o clima é tipo tropical quente e úmido (Af), em que, praticamente não há estação seca

Durante o período experimental foram registradas, no interior da casa de vegetação, as seguintes temperaturas: máxima de $37,9{ }^{\circ} \mathrm{C}$; mínima de $21,8^{\circ} \mathrm{C}$; média de $26,8{ }^{\circ} \mathrm{C}$ e umidade relativa do ar de $74 \%$.

A cultura utilizada foi a alface crespa "Robusta" cultivada em sistema hidropônico do tipo DFT (Deep Flow Technique) adaptado, conforme descrição de Silva et al., (2016). A SN (solução nutritiva) empregada no cultivo 
foi baseada na recomendação de Furlani (1998) para cultivo hidropônico de folhosas.

Os tratamentos consistiram na interação de duas qualidades de água caracterizadas em termos de condutividade elétrica da água (CEa). A água classificada como doce foi proveniente de abastecimento local (CEa 0,3 $\mathrm{dS} \mathrm{m}^{-1}$ ); a água salobra foi obtida a partir da adição de cloreto de sódio $(\mathrm{NaCl})$ na água de abastecimento local, atingindo CEa de 5,3 dS $\mathrm{m}^{-1}$, e três frequências de recirculação da solução nutritiva: 0,25 h; 2 e 4 h.

A interação foi arranjada em delineamento aleatorizado em cinco blocos, com uma repetição por bloco, totalizando 30 parcelas experimentais cada uma representando uma unidade de um sistema hidropônico DFT adaptado. A disponibilidade de solução nutritiva para cada planta foi de 3,4 L.

Ao longo do experimento, foram feitas reposições de nutrientes sempre que a condutividade elétrica da solução nutritiva (CEsol) do tratamento considerado testemunha (água doce $\mathrm{x}$ 0,25h) reduziu $25 \%$ do valor inicial. A reposição, quando necessária, foi realizada em todos os tratamentos. Também foram realizadas correções de $\mathrm{pH}$, procurando manter o mesmo numa faixa entre 5,5 e 6,5.

A análise sensorial foi realizada com plantas colhidas aos 28 dias após o transplantio (DAT). Participaram da análise 26 provadores, do sexo feminino e masculino com idade entre 20 e 60 anos, que avaliaram seis amostras cada um, as amostras se referiam aos seis tratamentos testados, aos quais foram denominados como: T1(DFT, 0,25 h, CEa 0,3dS m $\mathrm{d}^{-1}$ ); T2 (DFT, 0,25 h, CEa 5,3 dS m ${ }^{-1}$ ); T3(DFT, 2h, CEa 0,3 dS m ${ }^{-1}$ ), T4 (DFT, 2h, CEa 5,3 dS m-1), T5 (DFT, 4h, CEa 0,3 dS m$\left.{ }^{1}\right)$ e T6 (DFT, 4h, CEa 5,3 dS m ${ }^{-1}$ ).

Para o teste sensorial, as amostras foram codificadas com números de três dígitos e aleatorizadas, e logo após, servidas para os provadores, que foram instruídos a atribuírem uma nota para a avaliação, que deveria variar de 1 a 9, sendo: 1-desgostei muitíssimo; 2desgostei muito; 3- desgostei moderadamente;
4- desgostei ligeiramente; 5- nem gostei/ nem desgostei; 6- gostei ligeiramente; 7- gostei moderadamente; 8- gostei muito; 9- gostei muitíssimo. Nessa escala hedônica o provador deveria informar o quanto gostou ou desgostou de cada amostra em relação às variáveis: Sabor, Textura (crocância), Aparência e Impressão Global. Também foi questionado na mesma ficha de avaliaçãoo quanto os provadores estariam dispostos consumirem o produto avaliado.

Os dados da análise sensorial foram analisados por meio de Análise de Componentes Principais (ACP) feita a partir do software R v 3.3.1 (Pagés; Husson, 2001). A análise consiste essencialmente em transformar as n-variáveis originais em um novo conjunto de variáveis. As variáveis foram padronizadas segundo a Equação 1:

$$
Z=\frac{x-\bar{x}}{s}
$$

em que: $Z$ é o valor padronizado de $\mathrm{x}$; $\bar{x}$ é a média da característica;s é o respectivo desviopadrão.

O primeiro componente principal (Dim1) explica o maior percentual da variância total. O segundo componente principal (Dim2) explica a segunda maior percentagem e assim por diante, até que toda a variância é explicada, totalizando $100 \%$. Para tanto, é interessante saber a incerteza do meio de projeção de pontos no desenho fatorial. Conforme sugerido por Saporta e Hatabian (1986), a construção de regiões de confiança na análise de fatores é dada pela Equação 2. O i-ésimo componente principal é dado pela Equação 2:

$$
P C_{i}=e_{i}^{t} x=e_{i 1} x_{1}+e_{i 2} x_{2}+\ldots+e_{i p} x_{p}
$$

em que: $e_{i p}$ é o $p$-ésimoautovetor; $x$ é o $p-$ ésimo valor da variável original.

\section{RESULTADOS E DISCUSSÃO}


A Figura 1 apresenta o diagrama da análise de componentes principais
(ACP) feito a partir das médias dos cincos parâmetros da análise sensorial utilizados.

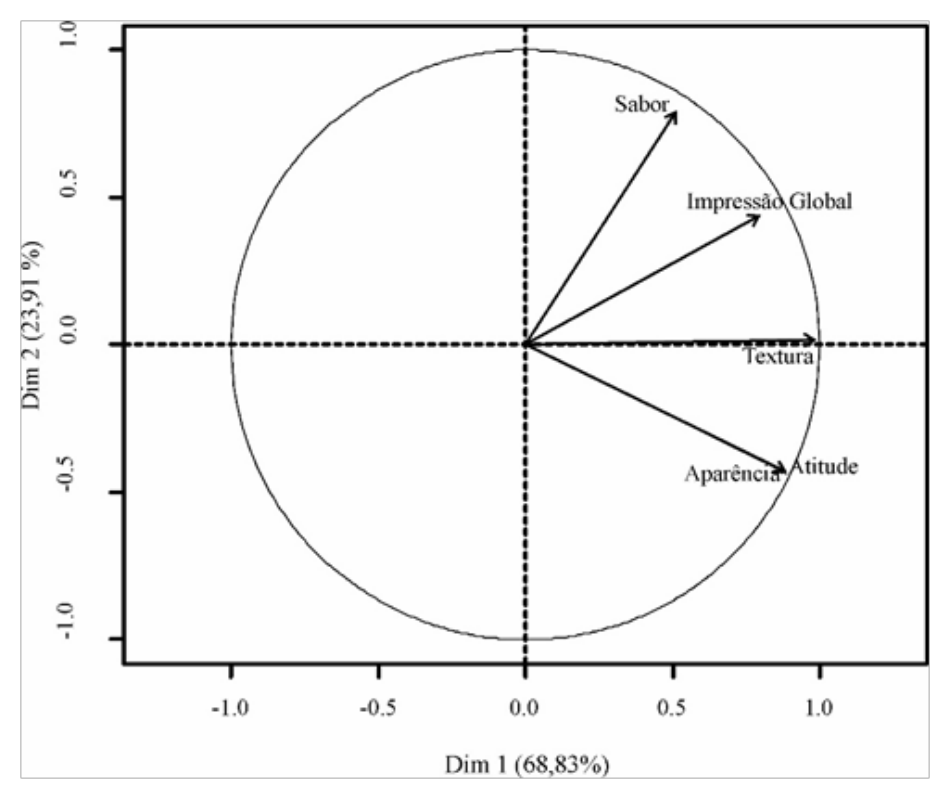

Figura 1. Análise de componentes principais para os atributos avaliados na análise sensorial de alface crespa "Robusta" submetida aos seguintes tratamentos: T1 (DFT, 0,25 h, CEa 0,3 dS m ${ }^{-1}$ ); T2 (DFT, 0,25 h, CEa 5,3 $\mathrm{dS} \mathrm{m} \mathrm{m}^{-1}$ ); T3 (DFT, 2h, CEa 0,3 dS m ${ }^{-1}$ ), T4 (DFT, 2h, CEa 5,3 dS m $\mathrm{m}^{-1}$ ), T5 (DFT, 4h, CEa 0,3 $\mathrm{dS} \mathrm{m}^{-1}$ ) e T6 (DFT, 4h, CEa 5,3 dS m

O diagrama da Figura 1 sugere que os dois primeiros eixos na análise de componentes principais (ACP) resumem bem a análise sensorial dos seis tratamentos. $\mathrm{Na}$ Tabela 1 é possível verificar mais precisamente que os dois primeiros eixos
(Dim1 e Dim2) têm respectivamente um percentual de inércia de 68,83 e 23,91 \%, o que juntos dão um percentual acumulado de 92,74\%. Assim, a análise de componentes principais permite recuperar $92,74 \%$ das informações contidas nas avaliações.

Tabela 1. Percentuais de inércia dos eixos da análise de componentes principais (ACP) da análise sensorial de alface crespa "Robusta"

\begin{tabular}{llll}
\hline Eixos & Dim 1 & Dim 2 & Dim 3 \\
\hline \% Individual & 68,83 & 23,91 & 7,26 \\
\% Acumulado & 68,83 & 92,74 & 100 \\
\hline
\end{tabular}

*Dim- dimensão

Existe uma forte correlação entre a aparência do produto e a atitude do consumidor (poder de compra/consumo). Esses dois fatores juntos se opõem quanto ao sabor e à impressão global para cada tratamento. O parâmetro textura se destaca dos demais, tendo assim, melhor aceitação por parte do consumidor, sendo bem representado pelo Dim1, o que indica que $68,83 \%$ das informações contidas na análise sensorial são bem representadas por esse parâmetro.

Os níveis de salinidade não comprometeram a aparência da alface em qualquer um dos tratamentos avaliados. Tal atributo fez com que os provadores demonstrassem interesse em consumir o produto, levando-se a concluir que os consumidores da alface fresca compram em função da aparência e da qualidade textural e provavelmente repitam sua compra em função da satisfação com o sabor.

Os resultados obtidos nesse estudo estão de acordo com os obtidos por Paulus et al., (2012) em análise sensorial de alface cultivado 
em diferentes níveis de salinidade da água, uma vez que os níveis de salinidade utilizados pelos autores não interferiram na aceitação da alface pelo consumidor.

A Figura 2 representa a distinção de grupos em função da ACP para cada tratamento. A alface cultivada com água doce e frequência de 0,25 h (T1) e a alface cultivada com águas salobras na mesma frequência (T2) apresentaram o mesmo perfil sensorial de acordo com os provadores, enquanto que a alface cultivada em água salobra com frequência de 4h (T6) não diferiu da alface cultivada com água doce e frequência de 0,25 h (T1). Portanto, apesar de não ter ocorrido diferenças entre os tratamentos avaliados, foi possível perceber que o consumidor preza muito pela aparência da alface no momento do consumo, acredita-se que isso pode estar relacionado ao fato de que as folhas de alface avaliadas no presente estudo não terem apresentado injúrias por deficiências nutricionais ou por efeito de íons tóxicos. No que diz respeito ao aspecto visual isso contribui para uma maior aceitação do produto.

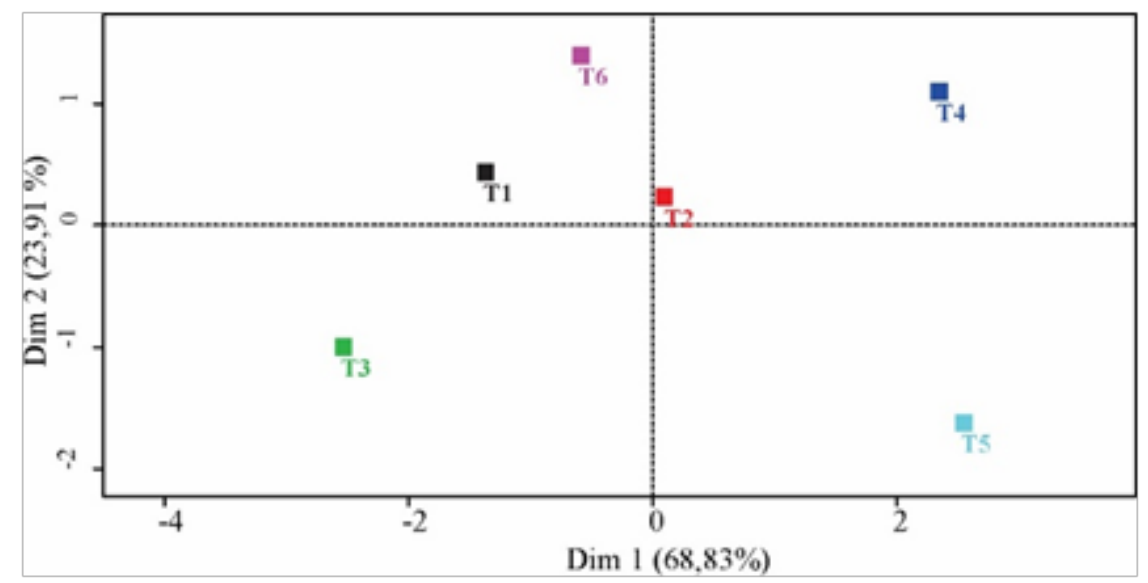

Figura 2. Distinção entre os tratamentos T1 (DFT, 0,25 h, CEa 0,3 dS m ); T2 (DFT, 0,25 h, CEa 5,3 dS m

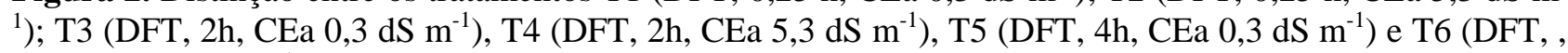
4h, CEa 5,3 dS m m $^{-1}$ ) em função da análise de componentes principais (ACP) da análise sensorial da alface crespa "Robusta"

\section{CONCLUSÕES}

O tipo de água e a frequência de recirculação da solução nutritiva não interferiu na aceitação da alface produzida em sistema hidropônico DFT. Os consumidores de alface comprariam em função da aparência e da textura do produto.

\section{AGRADECIMENTOS}

À Coordenadoria de Aperfeiçoamento do Pessoal de Nível Superior (CAPES), pela concessão da bolsa de estudos para o primeiro autor; ao Conselho Nacional de Desenvolvimento Científico e Tecnológico (CNPq),a Fundação de Amparo à Pesquisa do Estado da Bahia (FAPESB) e ao Instituto
Nacional de Ciência e Tecnologia em Salinidade (INCTSal),pelo apoio financeiro a pesquisa.

\section{REFERÊNCIAS}

ALVARES, C.A.; STAPE, J.L.; SENTELHAS, P.C.; GONÇALVES, J.L.M., SPAROVEK, G. Koppen's climate classification map for Brazil. Meteorologische Zeitschrift, v. 22, p. 711728, 2013.

COVA, A.M.W.; FREITAS, F.T.O.; VIANA, P.C.; RAFAEL, M.R.S.; AZEVEDO NETO, A.D.; SOARES, T.M. Content of inorganic solutes in lettuce grown with brackish water in different hydroponic systems. Revista 
Brasileira de Engenharia Agrícola e Ambiental, v. 21, p. 150-155, 2017.

FURLANI, P.R. Instrução para o cultivo de hortaliça de folha pela técnica de hidroponiaNFT. Campinas: Instituto Agronômico. Boletim Técnico, 168: 30p, 1998.

KRAUSE-SAKATE, R.; FIRMINO, A. C.; JADÃO, A. S.; PAVAN, M. A.; SILVA, N.; HANAI, S. M.; ANBO, R. H.; NIETZSCHE, T. Ocorrência generalizada do Lettuce mottle vírus em três regiões produtoras de alface do Estado de São Paulo. Summa Phytopathologica, v. 34, n. 1, p. 88-89, 2008.

PAGÉS J., HUSSON F.. Inter-laboratory comparison of sensory profiles. Methodology and results Food Quality and Preference, v. 12. p. 297-309, 2001.
PAULUS, D.; DOURADO NETO, D.; PAULUS, E. Análise sensorial, teores de nitrato e de nutrientes de alface cultivada em hidroponia sob águas salinas. Horticultura Brasileira, v.30, p.18-25, 2012.

SAPORTA, G., HATABIAN, G. Régions de confiance en analyse factorielle.[Confidence regions in factor analysis]. In: Diday, E. (ed.) Data analysis and informatics.Amsterdam, Elsevier. p 499-508, 1986.

SILVA, M.G.; SOARES, T.M.; GHEYI, H.R.; OLIVEIRA, I.S.; SILVA FILHO, J.A.; CARMO, F.F. Frequency of recirculation of nutrient solution in hydroponic cultivation of coriander with brackish water. Revista Brasileira de Engenharia Agrícola e Ambiental, v. 20, p. 447-454, 2016. 